\title{
Trends in Antibiotic Resistance Patterns among Salmonella Enterica Species - A Six Year Study from Tertiary Care Centre
}

\author{
Ashish William*, Aroma Oberoi, Divya D'souza, Arpit Oberoi
}

Christian Medical College \& Hospital, Ludhiana, India

DOI: $\underline{10.36347 / \text { sjams.2020.v08i09.018 }}$

| Received: 06.09.2020 | Accepted: 14.09.2020 | Published: 17.09.2020

*Corresponding author: Ashish William

Abstract

Original Research Article

Background: Typhoid remains a global public health problem with a higher burden in low- and middle-income countries (LMICs) due to poverty, limited access to safe water and unhygienic practices. The prevalence of Multidrugresistant strains has been responsible for outbreaks in India. In recent years, there has been an increase in fluoroquinolones resistance because of which ciprofloxacin is no longer the empirical choice of treatment in our country. Objectives: The aim of the study is to know the prevalence of enteric fever associated with Salmonella enterica and determine its antimicrobial susceptibility in a tertiary care hospital. Methods: This is a retrospective study conducted in the Department of Microbiology, CMC and Hospital, Ludhiana for 6 years. All the culture positive enteric fever cases were included in the study. Antimicrobial susceptibility was done as per corresponding CLSI guidelines. Results: A total of 918 strains of Salmonella species- Salmonella typhi $802(87.36 \%)$ and Salmonella paratyphi A $116(12.63 \%)$ were isolated from the blood cultures. Antimicrobial susceptibility for chloramphenicol, ampicillin and co trimoxazole was found to be $94 \%, 97 \%, 100 \%$ for S. Typhi and 94.63\%, 96.64\% and $100 \%$ for S. Paratyphi A, respectively. There is a continuous increase in Ciprofloxacin minimum inhibitory concentration values over the time. Majority of Salmonella isolates were nalidixic acid resistant. Although the rate of MDR Salmonella strains was nil, their reduced susceptibility to fluoroquinolones has restricted their routine empirical use. Conclusions: Enteric fever continues to cause significant morbidity due to delayed diagnosis, inadequate treatment and worsening drug resistance in India and beyond. There has been a reported decline in MDR with a parallel increase in decreased ciprofloxacin susceptibility among S. Typhi. Third generation cephalosporins are the safest choice for empirical use as ampicillin, chloramphenicol or cotrimoxazole are less likely preferred because of longer duration of therapy, threat of re-emergence of resistance, side effects and higher relapse rates. Judicious use of these antibiotics is mandatory to prevent emergence of resistant strains.

Keywords: Antimicrobial Resistance, Enteric Fever, Salmonella Paratyphi A, Salmonella Typhi, MDR, DCS.

Copyright @ 2020: This is an open-access article distributed under the terms of the Creative Commons Attribution license which permits unrestricted use, distribution, and reproduction in any medium for non-commercial use (NonCommercial, or CC-BY-NC) provided the original author and source are credited.

\section{INTRODUCTION}

Enteric fever (Typhoid) is community-acquired systemic infection which continues to be a public health problem in developing countries which is predominantly caused by Salmonella enterica serovar Typhi and also to a lesser extent by S. Paratyphi A. Humans is the only reservoir for these organisms. The common sources of infection are the stool and urine of infected persons, with the important vehicles being contaminated water, food and flies. The causative agent is either waterborne or foodborne for this gastrointestinal infection. The onset and severity of the disease mainly depends on the virulence of the organism and the infective dose[1]. Despite progress in controlling enteric fever in several parts of the world, it remains an important public health burden in South Asia. The incidence is estimated to be over 100 per
100000 population. Around seven million people are affected each year in South Asia with about 75000 deaths. However, these figures are likely to be an underestimate because of limitations in population based surveillance systems and reliable diagnostic methods [2]. As per the WHO guidelines [3], ciprofloxacin or ofloxacin is recommended for fully sensitive typhoid cases; alternatively, chloramphenicol, amoxicillin and co-trimoxazole are also recommended. However, the following two categories of drug resistance have been developed: resistance to antibiotics such as chloramphenicol, ampicillin and trimethoprimsulphamethoxazole [multidrug resistance (MDR) strains] and resistance to the fluoroquinolone drugs. For the MDR cases, ciprofloxacin or ofloxacin or cefixime or azithromycin or cefotaxime needs to be used. In case 
of quinolone resistance, azithromycin, rocephin or cefotaxime is recommended [3].

Azithromycin remains an effective oral option. However, given its wide use in a variety of clinical presentations, including suspected typhoid fever and respiratory infections, there is concern about potential emergence of resistant strains [4]. Third generation cephalosporins such as ceftriaxone and cefixime are increasingly used, with very low resistance reported to these drugs until recently [5]. Since 2016, outbreaks of extensively drug resistant $S$ Typhi strains that are resistant to ceftriaxone and cefixime have been reported in parts of Pakistan [15]. This severely limits the antimicrobial treatment options, and salvage therapy with intravenous carbapenems may be needed [6]. These drugs are expensive and often inaccessible in low resource settings.

MDR typhoid has become a major concern in India, with chloramphenicol resistance outbreak in 1972 followed by amoxicillin, co-trimoxazole and chloramphenicol by the 1990s. Ciprofloxacin resistance was developed in the late 1990s [7]. Currently; physicians prescribe azithromycin or cefixime for uncomplicated cases and ceftriaxone in intravenous therapy as per the National Treatment Guidelines for Antimicrobial Use in Infectious Diseases released by the National Centre for Disease Control. However, emerging resistance leading to more disease severity, morbidity and mortality emphasizes careful monitoring, surveillance and reporting of the cases to avoid last-line antimicrobials in therapy [7].

Combination therapies of azithromycin with ceftriaxone or cefixime and recycling of older antimicrobials have been debatable for quite some time. Regarding the policy framework, the National Policy for Containment of Antimicrobial Resistance was initiated by the Government of India in 2011[8], followed by the Chennai Declaration in 2012, and a five-year plan to implement antimicrobial stewardship remained a significant initiative. In 2014, Schedule H1 was introduced to restrict the sale of third-generation and beyond antibiotics without a prescription leaving aside, azithromycin and ciprofloxacin. Hence, infectious disease specialists and accredited microbiology laboratories hold serious promise for the effective control of MDR challenges [8].

\section{AIM}

This study was intended to determine the spectrum of Salmonella enteric serovars isolated from the blood culture of the patients suffering from enteric fever and their antibiotic susceptibility pattern to commonly used antibiotics in a community based tertiary care teaching hospital in Punjab.

\section{MeTHODS}

It was a retrospective ( 5 year) study for 6 years in the Department of Microbiology at Christian Medical College \& Hospital, Ludhiana. The samples which came by BACTEC (9120 and 9050) automated culture system were included as part of blood cultures; Becton Dickinson were included in the study. The diagnosis of enteric fever was usually done by using standard blood culture protocols and biochemical tests for identification were followed. The confirmation of the Isolates was being performed by using specific Salmonella antisera (DENKA SEIKEN CO., LTD. Tokyo, Japan) as a part of agglutination for serovar determination. Antibiotic susceptibility testing was performed by modified Kirby-Bauer disc diffusion test and minimum inhibitory concentrations (MICs) were determined using the the MicroScanWalkAway 96 system according to the Clinical and Laboratory Standards Institute guidelines for the corresponding year of isolation. The antibiotic discs for Antimicrobial susceptibility testing includes Ampicillin (10 $\mu \mathrm{g})$, chloramphenicol $(30 \mu \mathrm{g})$, co-trimoxazole (1.25/23.75 $\mu \mathrm{g})$, ciprofloxacin $(5 \mu \mathrm{g})$, ofloxacin $(5 \mu \mathrm{g})$, nalidixic acid $(30 \mu \mathrm{g})$, amikacin $(10 \mu \mathrm{g})$, gentamycin $(10 \mu \mathrm{g})$, cefotaxime $(30 \mu \mathrm{g})$,ceftriaxone $(30 \mu \mathrm{g})$, cefperazone $(75 \mu \mathrm{g})$, ceftazidime $(30 \mu \mathrm{g})$, piptaz $(100 / 10 \mu \mathrm{g})$, cefoperazone/sal $(30 / 10 \mu \mathrm{g})$, azithromycin $(15 \mu \mathrm{g})\left(\mathrm{Hi}^{-}\right.$ media Laboratories Ltd, Mumbai, India). MIC for ofloxacin was determined in the strains after the recommendation of these antimicrobial agents for enteric fever in 2013[8]. MIC for azithromycin was determined in the strains isolated after 2015 when the breakpoints were defined by CLSI [9].

The quality controls used for the antimicrobial susceptibility testing were Escherichia coli ATCC 25922 and Staphylococcus aureus ATCC 25923. The detection of extended spectrum beta-lactamases (ESBL) was also done for all Salmonella isolates.

The results are based on observations of the data and statistical analysis was not done for the study.

\section{RESULTS}

Out of total of 52,100 blood cultures received in the hospital, $918(1.76 \%)$ were culture positive for typhoidal salmonellae. The predominant serotype obtained was S. Typhi 802(87.36\%) followed by Salmonella paratyphi A 116 (12.63\%) shown in Figure 1. The age-wise distribution showed that $77(8.4 \%)$ cases occurred in <5 years age group, $512(55.8 \%)$ cases in 5-18 years age group and $326(35.8 \%)$ were found in $>18$ years of age, shown in Figure 2. In our study it was also observed that culture positive patients include 693 male (76\%) and 225 female (25\%) and the male to female ratio is 3.1:1 Figure 1. Typhoid fever cases occurred in all months throughout the year, however they peaked during the months of May- 


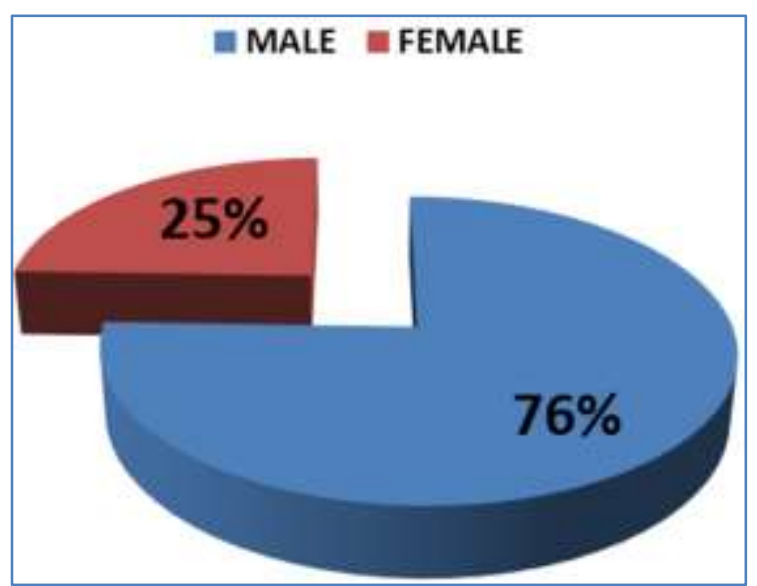

Fig-1: Gender wise Distribution of Typhoid Positive Patients

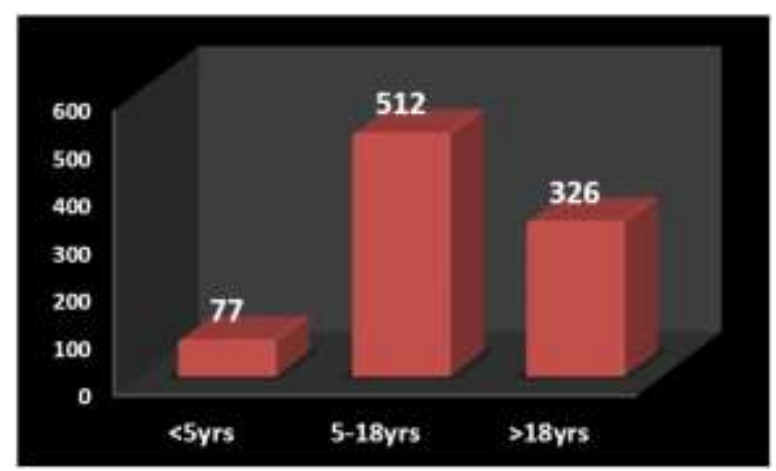

Fig-2: Age wise Distribution of Typhoid Positive Pateints (N=918)

S. typhi and S. Paratyphi A were both found to be $100 \%$ susceptible to Co-trimoxazole, amikacin and gentamycin. S. typhi showed higher susceptibility to Azithromycin (98.89\%) and Ampicillin (98.48\%) than S. Paratyphi A $97.99 \%$ and $96.64 \%$ respectively. Whereas resistance to Chloramphenicol was the highest among all antibiotics, it was found to be more in S.typhi isolates (8\%) as compared to S. Paratyphi A (5.70\%). Resistance to nalidixic (NA) acid was found to be high; it has been rising each year with an average resistance of $98.40 \%$ and was $100 \%$ in $2017 \& 2018$. There has been observed a continuous increase in Ciprofloxacin minimum inhibitory concentration (MIC) values over the time. A positive correlation was observed between reduced ciprofloxacin susceptibility and Nalidixic Acid resistance in all the isolates. S. Paratyphi A strains showed higher rate $(99.13 \%)$ of NA resistance than S. Typhi (98.4\%).The antibiotic susceptibility is shown in Table 1.

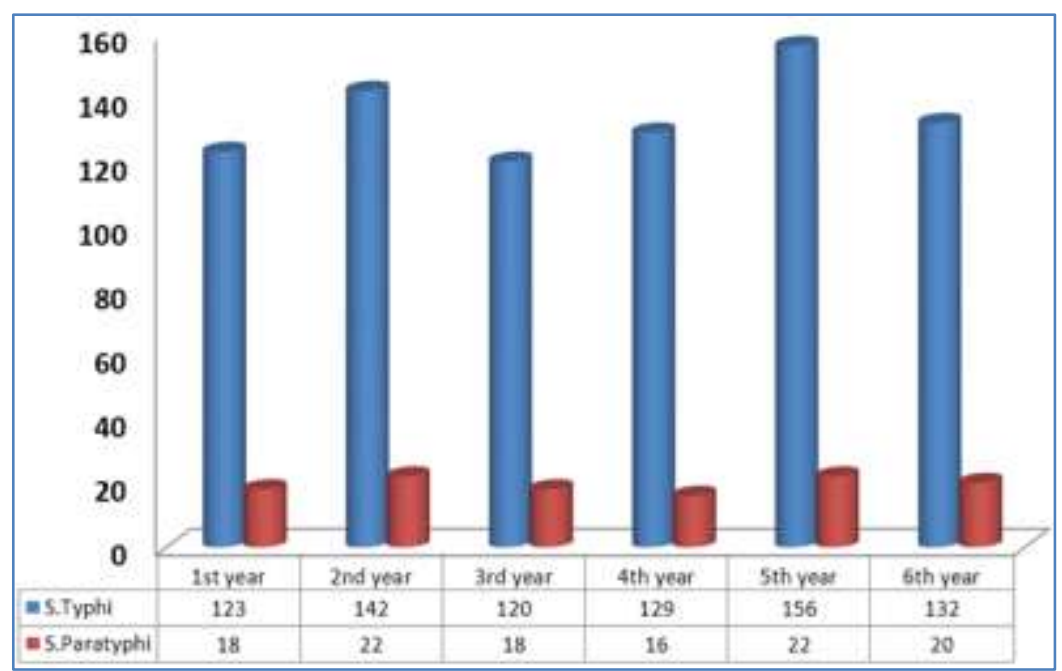

Fig-3: Year Wise Distribution of Salmonella Isolate (S.Typhi N=802 and S.Paratyphi N=116) 
Table-1: Antimicrobial Susceptibility of Salmonella Species (S.Typhi N=802 and S.Paratyphi N=116)

\begin{tabular}{|c|c|c|c|c|c|c|}
\hline Antibiotics & \multicolumn{3}{|c|}{ S.typhi } & \multicolumn{3}{c|}{ S.paratyphi A } \\
\hline & S & I & R & S & I & R \\
\hline Co-trimoxazole & 802 & 0 & 0 & 116 & 0 & 0 \\
\hline Amikacin & 802 & 0 & 0 & 116 & 0 & 0 \\
\hline Gentamycin & 802 & 0 & 0 & 116 & 0 & 0 \\
\hline Ceftazidime & 802 & 0 & 0 & 114 & 1 & 1 \\
\hline Cefotaxime & 799 & 1 & 2 & 115 & 1 & 0 \\
\hline Cefoperazone & 799 & 0 & 3 & 116 & 0 & 0 \\
\hline Piptaz & 799 & 1 & 2 & 113 & 2 & 1 \\
\hline Cefo/sulb & 799 & 0 & 3 & 116 & 0 & 0 \\
\hline Ceftriaxone & 798 & 2 & 2 & 115 & 0 & 1 \\
\hline Azithromycin & 794 & 4 & 4 & 113 & 1 & 2 \\
\hline Ampicillin & 791 & 2 & 9 & 111 & 1 & 4 \\
\hline Nalidixic acid & 13 & 0 & 789 & 1 & 0 & 115 \\
\hline Ofloxacin & 13 & 0 & 789 & 1 & 0 & 115 \\
\hline Ciprofloxacin & 13 & 5 & 784 & 1 & 0 & 115 \\
\hline Chloramphenicol & 744 & 58 & 0 & 108 & 8 & 0 \\
\hline S: some,
\end{tabular}

$S$ : sensitive, $I$ : intermediate sensitive, $R$ : resistant.

The resistance pattern of Nalidixic Acid observed was seen to be almost similar to the Fluoroquinolones. There are no ESBL or multidrug-resistant strains in any Salmonella isolates.

In Salmonella Typhi the susceptibility to cephalosporins like ceftazidime (100\%), cefotaxime (99.6\%), cefoperazone $(99.6 \%$, and ceftriaxone $(99.5 \%)$ is quite high. piptaz,Cefo/sulb (99.59\%). wheras Salmonella Paratyphi A isolated were 100\% susceptible to cefoperazone, Cefo/sal, followed by cefotaxime and ceftriaxone $(99.33 \%)$, ceftazidime $(98.66 \%)$ and piptaz $(97.99 \%)$. The minimum inhibitory concentration (MIC) 50 and $\mathrm{MIC}_{90}$ values $(0.032$ to 0.94 in S. Typhi followed by $0.019-0.75$ ) for ceftriaxone was observed to increase over the time which indicates the tendency of ceftriaxone moving towards the resistance over the years.However more data is needed for the resistance pattern.

\section{DiSCUSSION}

Though enteric fever occurs at any age the higher incidence is observed in children who state the active transmission in a community [10]. There is an estimation of prevalence of laboratory-confirmed enteric fever among individuals which is seven per cent for Salmonella Typhi and 0.9 per cent for Salmonella Paratyphi A with the highest incidence in children as per meta-analysis data on the burden of typhoid and paratyphoid fever in India $[11,12]$. In our study also the results are similar as the greater population of Enteric fever is observed in children between 5-18 years (5-18 yrs: $55.8 \%$ and >18 yrs: $35.8 \%$ as seen in Figure 2).

The third-generation cephalosporins are presently the drug of choice for the treatment of typhoid fever. The clinical studies on efficacy are available for parenteral ceftriaxone only (not oral cefixime) and increasing MIC to ceftriaxone is a cause of concern [13]. There is similar result for ceftriaxone in our study with increasing MIC over a period of 5 years with increase in MIC over the years.

In our study the nalidixic acid resistance was seen to be $98.4 \%$. It was similar to a study for trends over 3 years in chandigarh which showed nalidixic acid resistant $S$. Typhi (NARST) increased from $90.2 \%$ to $100 \%$ [14]. The increasing resistance to fluoroquinolones observed in the present study is perhaps a direct consequence of their indiscriminate prescription not only for typhoid fever but also for other infections. It is also associated with a concomitant reemergence of chloramphenicol susceptibility attributed to its restricted use, which resulted in the withdrawal of selection pressure.

Besides fluoroquinolones, the overall susceptibility of Salmonella isolates to chloramphenicol was found to be lowest $(92.7 \%$ for S. Typhi and $93.1 \%$ for S. Paratyphi). Susceptibility of Salmonella isolates to other first-line drugs, that is, ampicillin, cotrimoxazole, and azithromycin, was also excellent. The reason for susceptibility to remerge again is probably due to the decreased usage of first line antibiotic in the treatment of enteric fever and even in other infections. Due to irrational use of antibiotics, the rate of drug resistance among bacteria is increasing and the situation is worse in developing countries. So antibiotics should be used only on the basis of culture and sensitivity report [15].

Increased use of cephalosporin or azithromycin in treating fluoroquinolonenons usceptible S. Typhi may lead to a rise in cephalosporin resistance or azithromycin treatment failure. In the era of MDR, combination therapy could be an alternative for treating enteric fever cases. 


\section{CONCLUSION}

Before the fixed-dose combinations are prescribed, second antibiotic should be added only on clinical judgment in selected cases. Furthermore, there is a need to strengthen preventive measures like safe water supply and by developing new vaccines that are effective against both $S$. Typhi and $S$. Paratyphi A as there is no new drug is in the horizon. The conventional first-line drugs along with the third generation cephalosporins and azithromycin can be used as empiric therapy for treatment of enteric fever in our setting. Multi-centered studies covering wide geographical area and large population are required to generate more significant data regarding the susceptibility of the Salmonella spp. toward ampicillin, co-trimoxazole and chloramphenicol and to determine the possibility of using these drugs for empirical therapy for treatment of enteric fever.

\section{REFERENCES}

1. Miller SI, Pegues DA. Salmonella species, including Salmonella Typhi. In: Mandell GL, Bennett JE, Dolin R, editors. Principles and practice of infectious diseases, Vol. 5. Philadelphia: Churchill Livingstone; 2000: 2344-63.

2. Mogasale V, Maskery B, Ochiai RL, Lee JS, Mogasale VV, Ramani E, Kim YE, Park JK, Wierzba TF. Burden of typhoid fever in lowincome and middle-income countries: a systematic, literature-based update with risk-factor adjustment. The Lancet Global health. 2014 Oct 1;2(10):e57080.

3. World Health Organization. Guidelines for the management of the typhoid fever; 2011.

4. Crump JA, Sjölund-Karlsson M, Gordon MA, Parry CM. Epidemiology, clinical presentation, laboratory diagnosis, antimicrobial resistance, and antimicrobial management of invasive salmonella infections.

5. Balaji V, Kapil A, Shastri J, Pragasam AK, Gole G, Choudhari S, Kang G, John J. Longitudinal typhoid fever trends in India from 2000 to 2015. The American journal of tropical medicine and hygiene. 2018 Sep 6;99(3_Suppl):34-40.

6. Klemm EJ, Shakoor S, Page AJ. Emergence of an Extensively Drug-Resistant Salmonella enterica Serovar Typhi Clone Harboring a Promiscuous Plasmid Encoding Resistance to
Fluoroquinolones and Third-Generation Cephalosporins.2018.

7. Bharmoria A, Shukla A, Sharma K. Typhoid fever as a challenge for developing countries and elusive diagnostic approaches available for the enteric fever. Int J Vaccine Res 2017; 2: 1-16.

8. Divyashree S, Nabarro LE, Veeraraghavan B, Rupali P. Enteric fever in India: Current scenario and future directions. Trop Med Int Health. 2016; 21: 1255-62.

9. Clinical and Laboratory Standards Institute (CLSI). 2013. Performance standards for antimicrobial susceptibility testing; approved standard; 23rd informational supplement. CLSI document M100S23. Clinical and Laboratory Standards Institute (CLSI). 2015. Performance standards for antimicrobial susceptibility testing; approved standard; 25th informational supplement. CLSI document M100-S25.

10. John J, Van Aart CJ, Grassly NC. The burden of typhoid and paratyphoid in India: Systematic review and meta-analysis. PLoS Negl Trop Dis. 2016; 10:e0004616.

11. Crump JA, Sjölund-Karlsson M, Gordon MA, Parry CM. Epidemiology, clinical presentation, laboratory diagnosis, antimicrobial resistance, and antimicrobial management of invasive Salmonella infections. Clin Microbiol Rev. 2015; 28:901-37.

12. Arndt MB, Mosites EM, Tian M, Forouzanfar MH, Mokhdad AH, Meller M. Estimating the burden of paratyphoid a in Asia and Africa. PLoS Negl Trop Dis. 2014; 8:e2925.

13. Dahiya S, Sharma P, Kumari B, Pandey S, Malik R, Manral N. Characterisation of antimicrobial resistance in Salmonellae during 2014-2015 from four centres across India: An ICMR antimicrobial resistance surveillance network report. Indian J Med Microbiol. 2017; 35:61-8.

14. Gupta V, Singla N, Bansal N, Kaistha N, Chander J. Trends in the antibiotic resistance patterns of enteric Fever isolates - a three year report from a tertiary care centre. Malays J Med Sci. 2013;20(4):71-5.

15. Shrestha KL, Pant ND, Bhandari R, Khatri S, Shrestha B, Lekhak B. Re-emergence of the susceptibility of the Salmonella spp. isolated from blood samples to conventional first line antibiotics. Antimicrob Resist Infect Control. 2016;5:22 